history the idea not only of sequence, but of the lapse of how long before and how long after, can be mastered. The attempt to lathom the geological past with our short historical sounding line has up to the present time resulted merely in estimates, varying according to the assumed basis in each by thousands of centuries, that have been about as valuable in geological theory as Archbishop Usher's chronology has been found in Jiblical criticism. The problem is hedged in by innumerable difficulties, which cannot be overcome in the present state of science.

W. Boyd Dawkins

\section{On the Diffraction Spectrum and Wave Lengths}

THE letter which I wrote about the diffraction spectrun has called forth several inquiries. I have been asked how it is that in that spectrum the position of a line depends only on its wave-length, and I may take the opportunity of answering these questions through your columns. First, however, let me state that the numbers given in the table in my letter of February 9 are copied, including the obvious errors, verbatim from the translation of Mossotti's memoir in vol. v. of Taylor's "Scientific Memoirs." I attach no importance to such analogies. Any analogy between these lines and interdependent notes of music must, I expect, be entirely accidental. The latest experiments made by Mr. Lockyer have shown that the lines given ont by a gas vary according to temperature and pressure, and if these be caused by vibrations of the particles of the gas, whether atomic or molecular, the periods of these vibrations must be dependent on the temperature and pressure. A distinct numerical statement of this interdependence is a great desideratum in this part of science with a view of affording the materials for making and testing a mechanical theory of that interdependence. These are the great problems which demand our attention, as I take it, with respect to the fixed lines, and our ability to correlate light with other modes of force.

If a beam of homogeneous light be admitted through a small hole into a dark room, and fall on the opposite wall, it will illumirate only the portion of the wall directly opposite it. But if a series of fine parallel wires, or a fine grating be put across the hole, there will be seen ranged in a line perpendicular to the grating a series of nearly equal clistant spots, or rather streaks of light. This is because the wixes of the grating shut out those waves of light which at these points would destroy the other waves. To understand this more clearly, suppose $A a, B b, \& c$, $\& c$., to be a number of consecutive and equidistant points in the front of a wave coming through a small hole, Aa, \&c. These may, by the principle of Huyghens, be regarded each as the origin of a new wave, together having the same effect as the original

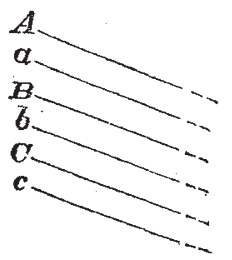

\section{7}

wave, all starting in the same phase. There is darkness at every point on an opposite wall, except directly opposite the hole, because of the interference of these elementary waves, which destroy one another everywhere except exactly in front. This is owing to the shortness of the waves and the number of sources. Let $\mathrm{P}$ be a point on the wall which we shall suppose sufficiently far from the hole in order that the lines $\mathrm{PA}, \mathrm{Pa}, \mathrm{PB}$, $\mathrm{Pb}, \& \mathrm{c}$, may be regarded as all sensibly parallel. On that account also each of these lines exceeds the one next it by the same amount. Now, generally, when the waves from A, a, \&c. arrive at $P$, the wave from $a$ will not entirely blot out that from $A$, but a residue will be left, which will be blotted out by other similar residues. But there will be certain points along the wall at which the waves will blot one another out in a peculiarly regular way. We shall suppose $\mathrm{P}$ to be one of these points, namely, that each of the distances A P, $a \mathrm{P}, \& \mathrm{c}$., exceeds the one next it by exactly some odd multiple of the length of half a wave-say in this instance half a wave's length. Then in this case the disturbance originating at $a$ will arrive at $\mathrm{P}$ exactly half a wave's length in actvance of that from $A$, and the wave from $A$ will be exactly blotted out, so to speak, by that from $a$; similarly that from 13 will be exactly blotted ont by that from $b$; and so on. So that if I were to put wires at $a, b, c$, \&c., so as to stop up the waves entering there, the waves from A, B, C, \&c., would fall at $P$ each undestroyed and assisting one another, each disturbance being an exact wave-length behind the other, and therefore all in the same phase. Thus light would be restored at $\mathrm{P}$. And similarly at other points wliere the difference between A P, $a$ P, \&c., is three, five, \&c., semi-wave-lengths. Light would be restored in the same way at no other places becaise the elementary waves do not at other points destroy one another in this peculiar alternative manner. Supposing $a, b, c, \& c_{\text {. }}$, to be the centres of the wires, A, B, C, \&c., to be the centres of the spaces between, a more refined consideration shows us that the position of $\mathrm{P}$ does not depend on the space $\mathrm{A} a$ being equal to the space $a \mathrm{~B}$, but only on the total length $\mathrm{A} \mathrm{B}$ (or $a b$ ). Hence we have a very accurate method of cletermining the wave-length from the position of $P$. For if $\theta$ be the angle between the line drawn from the hole straight forward in the direction of the ray and the line drawn to $P$, then the difference between A P and $B P$, which is a wave-length, is also $A B \sin \theta$. So that to get the wave-length all that we have to do is to measure $\theta$ and $A B$. A B may be very accurately got by ruling the grating on glass with a machine, and counting the whole number of rules given and the total space which they occupy. Adaptations of this method may be made to suit circumstances, as for instance the grating may be attached to the object glass of a telescope used for measuring the angle $\theta$. It is by such a method that the wave-lengths have been found for the principal fixed lines in the solar spectrum, and the wave-lengths of all other lines are determined from these by some formula or other which may best suit the views of the calculator; the constants of the formula being determined so as to constrain the formula to satisfy the truth at those fixed lines whose wave-lensths are got dixectly from the refraction spectrum. Uncertainty, therefore, prevails about all wave-lengths thus obtained, although, of course, the uncertainty must, from the method of calculation and the number of lines whose wave-lengths have been obtained from the diffraction spectrum, lie necessarily between narrow limits. These latter are the only wave-lengths, however, which have the recommendation of being due to direct observation; and the method of obtaining the wave-length by observation from the diffraction spectrum is one capable of such accuracy, that I have sometimes considered that the wave-length thus determined might be used as ari absolute and recoverable stanclard of linear measure.

Of course, the preceding investigation must not be considered to be perfectly exhaustive in giving the whole character of the phenomena, which are explained by a more refined investigation, in which the space between each wire is regarded, not as the source of one, but of an infinity of waves, so that the application of Huyghens' principle becomes rigidly correct. By this means the difference of the spaces occupied by the wire and the opening is found to have no effect on the position of $l^{2}$, but a certain effect on the brightness there, which, under certain circumstances, causes one of the spectra entirely to disappear : an experimental result which, being thus deducible from the theory of undulations, gives a striking proof of the power of that theory to account for phenomena.

Trinity College, Cambridge, March 2

\section{The Valuation of Liquid Town Sewage}

IF there is one thing that is more to be deprecated than another, it is the unnecessary importation of personalities into a scientific discussion, or indeed into any serious matter of business, and fortunately this is not now a common proceeding in this country. It was therefore with a feeling of unqualified disappointment that I read Dr. Paul's personal attack on myself in your columns.

Whenever I hear or read an opinion or statement which I believe to be erroneous, I endeavour to point out its fallacy, and shall always continue to do so. It is the undoubted right, as it is in some degree the duty, or every man to do this. But opinions may be criticised and condemned without any reflection on the man who holds them. This is all I did in Dr. Paul's case, and I certainly endeavoured to do it good-naturedly; at all events I not only made no reffection whatever on Dr. Paul's character, but I did not even mention his name. He had, in his article, hazarded farming, of which, so far as I know, he does not profess to have any personal knowledge or experience whatever. This opinion I knew from experience to be utterly erroncous, although 